\title{
Evaluating the role of botulinum toxin in the management of focal hypertonia in adults
}

\author{
Davina Richardson, Geoff Sheean, David Werring, Margi Desai, Susan Edwards, \\ Richard Greenwood, Alan Thompson
}

\begin{abstract}
Objectives-To investigate the effects of EMG guided botulinum toxin (BTX-A) on impairment and focal disability in adults presenting with focal hypertonia.

Methods-A prospective, randomised, double blind, placebo controlled, parallel group trial was carried out with standardised assessment before and at 3 week intervals until 12 weeks after injection, in patients with focal hypertonia affecting upper or lower limbs. Botulinum toxin or placebo was injected with EMG guidance after multidisciplinary assessment. The modified Ashworth scale of spasticity, percentage passive range of joint motion, subjective rating of problem severity, the Rivermead motor assessment scale, a timed 10 metre walk (lower limb patients), nine hole peg test (upper limb patients), and a modified goal attainment scale were used as outcome measures. The patients were 52 adults; 34 male, 18 female; mean age 40.31 , range $16-79$ years; mean duration of symptoms 35 months (range 3 months to 22 years). Diagnoses included cerebrovascular accidents (23), head injury (12), incomplete spinal cord injury (six), tumour (five), cerebral palsy (three), and anoxic episodes (three).
\end{abstract}

Results-For each variable an overall score for the treatment period was computed by summing the scores from the 3, 6, 9, and 12 week assessments. These overall scores were significantly better in the treated group for the Ashworth scale, percentage passive range of movement, Rivermead lower limb, and subjective rating of problem severity. The significant treatment effect on the Ashworth scale was seen on analysis of variance (ANOVA) at 3 weeks and the subjective rating of problem severity at 3 and 6 weeks. The goal attainment scale score in both groups was similar at 12 weeks.

Conclusion-Selective use of botulinum toxin to weaken muscles can lead to a reduction in resistance to passive movement about a distal limb joint. This allows for improvements in passive range of movement and focal disability, particularly in patients presenting with focal spasticity of the lower limb.

(F Neurol Neurosurg Psychiatry 2000;69:499-506)

Keywords: botulinum toxin type A (BTX-A); hypertonia; electromyography (EMG)
The resistance felt when moving a limb passively is termed hypertonia. Spasticity is defined as a velocity dependent increase in the tonic stretch reflex with exaggerated tendon jerks. ${ }^{1}$ It is now accepted that the exaggerated stretch reflex in a muscle is only partly responsible for hypertonia and that other positive features of the upper motor neuron syndrome, such as cocontraction, and biomechanical changes contribute significantly to the resistance to passive movement. ${ }^{23}$

When making decisions on methods of treatment and management of hypertonia it is useful to have some indication of the extent to which spasticity is responsible for the positioning of a limb and the resistance to movement. Electromyography identifies the presence of abnormal muscle activity. ${ }^{4}$ Recordings from specific muscles at rest with passive stretch give information about the resting state of the muscle and the stretch reflexes. Recordings from agonist and antagonist muscles simultaneously during movement may disclose abnormal cocontraction and may help gauge the voluntary strength of agonists.

Spasticity and the other positive features of the upper motor neuron syndrome can be targeted with botulinum toxin (BTX-A). This acts peripherally to block neuromuscular transmission by preventing the release of the neurotransmitter acetylcholine. ${ }^{5}$ It produces a partial chemical denervation of the injected muscle with subsequent weakness and atrophy.

The recent licensing of BTX-A for use in equinus deformity in cerebral palsy follows several years of investigation into the benefits of weakening spastic muscle in children. ${ }^{6-8} \mathrm{Com}-$ paratively little convincing evidence is available on its use in the management of adult limb spasticity. Several open labelled trials indicate that it is effective in reducing resistance to passive movement but sample sizes are small. ${ }^{9-11}$ To date there are only three randomised double blind placebo controlled trials. ${ }^{12-14}$ Simpson et al performed large numbers of pairwise comparisons between baseline and all subsequent time points without adjusting for multiple comparisons and the numbers in the treatment groups were less than $10 .{ }^{13}$ This raises doubts over the significance of the results, which showed reduction in spasticity but no functional gains. Burbaud et al used a cross over design to study the use of the toxin for equinus deformity of the foot raising the concern of an inadequate wash out period. ${ }^{12}$ Both these studies had selective patient groups with fixed dosing regimen, which does not reflect current clinical practice. Hesse et al had very few patients $(n=24$, randomised into four groups of 
six) limiting the interpretation of the results of this study. ${ }^{14}$ The lack of large double blind trials with more representative samples may reflect the inherently difficult nature of such studies, particularly in relation to recruiting patients, standardising injection procedure, measuring treatment outcomes, and evaluating results.

However, the evidence to date does at least suggest that BTX-A effectively reduces resistance to passive movement about a joint, which can influence the "static" consequences of hypertonia, such as pain, care delivery, and the resting posture of a limb. ${ }^{9-16}$ Similar to the experience with the introduction of baclofen, there is little conclusive evidence to show that reduction of the hypertonia results in improvements in active functional movements. Further blinded trials are needed to ascertain whether patients benefit functionally. In the present study we have used comprehensive multidisciplinary clinical assessment and detailed neurophysiological evaluation with needle EMG to guide treatment decisions and select patients who might benefit at the level of impairment and focal disability from weakening a muscle. The sample population included patients referred for evaluation of the use of focal botulinum toxin who met the inclusion critera. The doses were tailored for amount and location for each patient, reflecting current clinical practice.

\section{Patients and methods}

The study was a prospective, randomised, double blind, placebo controlled, parallel group trial.

Referrals were made to the specialist botulinum toxin clinic from various centres including rehabilitation units, physiotherapy departments, and specialist neurology clinics, on the basis that the treating therapists thought that the patient had potential to improve. The clinic team included a consultant neurologist, a consultant neurophysiologist, and a senior physiotherapist.

Identification of target muscles was made after comprehensive assessment, including information from the patient, carer, and treating therapists. Target muscles clinically thought to be responsible for the problem were sampled with needle EMG. Toxin was considered to be appropriate where stretch induced EMG activity was recorded (spasticity), when continuous resting activity was present (spastic dystonia), or if there was evidence of cocontraction. In some cases all three EMG patterns were present.

If a stretch response or other overactivity was not seen, other sites were sampled. Muscles were not injected if the tone increase was not accompanied by muscle overactivity and was thought to be mainly soft tissue in origin. In some cases, it was found that the hypertonia was due predominantly to biomechanical changes in the muscle rather than active muscle contraction. After the team decision to inject, according to the degree of overactivity and the identification of an achievable goal of intervention, an independent observer allocated the patient to control or treatment group with a list of placebo or treatment that was prepared by the hospital pharmacy using a latin square design. The injection material was then prepared accordingly in a separate room.

Injections of BTX-A (Botox, Allergan) or placebo were given via 21 gauge teflon coated needles. Vials of 100 units of toxin were diluted in $2 \mathrm{ml}$ saline. Placebo injections were performed using identical syringes containing $2 \mathrm{ml}$ saline only. The injection sites were identified with standard neurophysiological techniques. The doses and distribution of the injections were guided by the clinical and neurophysiological evaluation (including size of the stretch response and degree of resting activity). For example, if the presenting problem was an inability to straighten the elbow due to hypertonia, biceps, brachioradialis, and brachialis were examined to see the extent to which each was contributing to the flexed posture of the elbow and the doses targeted and titrated accordingly.

Inclusion criteria were the presence, for at least 2 months, of moderate to severe spasticity in focal muscle groups with a poor (defined clinically as failure to resolve the presenting problem) response to conventional physical and medical treatment. All patients had to be participating in an active rehabilitation and management programme. Exclusion criteria were the presence of fixed joint posture, progressive neuromuscular disease, previous injection of the BTX-A in the past 12 months into the same limb or 6 months into a separate limb, pregnancy, and those patients on anticoagulants whose clotting ratio (INR) exceeded 2.5.

Patients with various diagnoses were accepted into the trial, they were stratified into two groups: upper limb and lower limb presentation. Within these groups patients were then randomly allocated. No change in other medical treatment for spasticity was allowed during the course of the study.

\section{ASSESSMENTS}

Five to 10 days before clinic appointment each patient was assessed with a combination of measurements including standardised measures of disability and subjective rating scores. The primary outcome measure of impairment was the degree of resistance to passive movement of the target muscle group which was assessed using the modified Ashworth scale of spasticity. ${ }^{17}$ The passive range of movement at the target joint was measured using a standard hand held goniometer. ${ }^{18}$ To compare change between patients treated for different joints (which have different degrees of movement), the change in range was calculated as a percentage of the normal passive range of movement for each joint. For example a patients who was recorded as having 10 degrees of wrist extension from a position of neutral and full flexion ( 80 degrees) would be said to have $60 \%$ range of movement at that joint (full range of flexion=80 degrees; full range of extension $=70$ degrees; $90 / 150=60 \%$ ). 
Table 1 Exclusion criteria

\begin{tabular}{ll}
\hline Number of cases & Reason for not being suitable for treatment with BTX-A \\
\hline 3 & Previous injections \\
4 & Weakness around joint \\
7 & Biomechanical shortening of muscle \\
3 & Not a focal problem \\
3 & Problem identified was due to proximal difficulties not distal \\
2 & Transient increases in tone only \\
1 & Responding well to conventional treatment \\
\hline
\end{tabular}

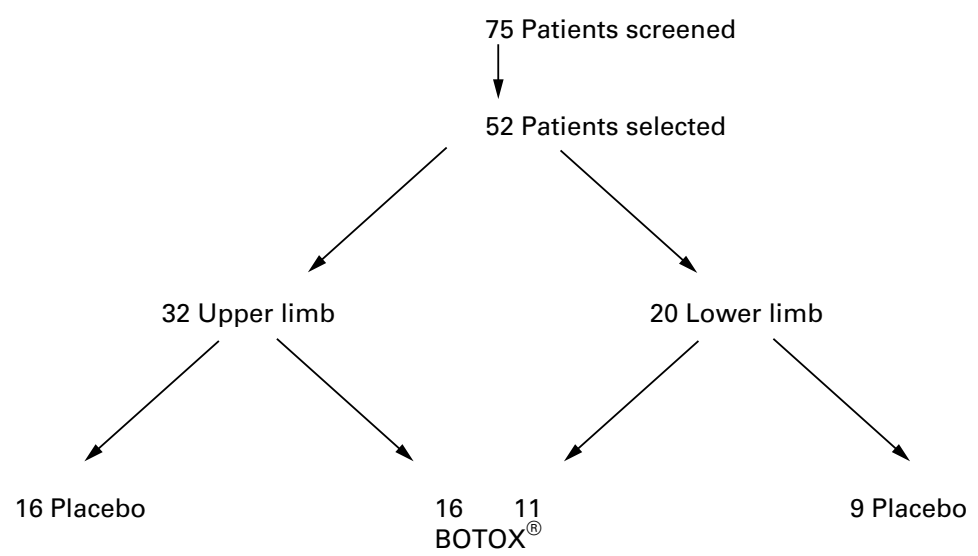

Figure 1 Scheme of study. tailed Mann-Whitney tests on these overall outcome scores were computed to provide an estimate of overall treatment effects. To try to follow the treatment effects across the trial, an analysis of variance (ANOVA) is required. As the raw data were unsuitable for conventional ANOVA, they were rank transformed before analysis and tested for normality. The statistical robustness of this approach has been repeatedly demonstrated. ${ }^{22}$ For the Ashworth scale, range of movement, Rivermead motor assessment scale, and the self rated problem scores, the data were ranked (with ties) for each patient separately over the five measurement points, with a rank of 5 being given to the patient's highest score. Under the null hypothesis of no effect across the time points of the study then, at each time point, the expected mean rank across patients in a group would be 3.0. If a set of scores increased monotonically with time then the mean ranks at earlier time points would be below 3 and at later time points would be above 3. (The ratings of change from baseline were ranked over the four measurement points after the baseline, with expected mean ranks of 2.5 under the null hypothesis.) The ranked variables were entered into a mixed design ANOVA, with factors of group (treatment $v$ placebo) and time (a repeated measures factor with five categorical levels, one for each of the five assessment time points between baseline and 12 weeks). Time was entered as a categorical variable. When a specific treatment effect was disclosed (by a significant group by time interaction), planned contrasts between successive timepoints (week $3 v$ baseline; week $6 v$ week 3; week $9 v$ week 6 ; week $12 v$ week 9) were carried out. These contrasts clarified when, and for how long, any treatment effect occurred. Other analyses used $\chi^{2}$ methods for contingency tables.

\section{Results}

Seventy five patients were screened over a 16 month period. Fifty two patients, 34 male; and 18 female, were recruited into the trial. The reasons for exclusion are documented in table 1. The mean age of the patients was 40.13 (range 16-79) years. The mean interval from injury to treatment was 35 months (range 3 months to 22 years). Twenty six patients had symptoms for over 12 months. Of the 52 recruited, 25 patients received placebo and 27 received active drug. A total of 32 upper limb patients (16 BTX-A; 16 placebo) and 20 lower limb patients (11 BTX-A; nine placebo) were recruited (fig 1). The groups were well matched for age, diagnosis, and duration since injury (table 2). The dependent variables were also well matched at baseline, although there was a weak trend for the treated and placebo groups to differ on the passive range of movement and Rivermead lower limb/trunk function before treatment (table 3).

In the upper limb group the mean number of muscles injected was 3.34 (range 1-10). The mean size of the dose distributed into the various muscles in the upper limb was 141.25 (SD 78.55) units (range 30-305). In the lower limb group the mean number of muscles injected 
Table 2 Comparison of demographic details

\begin{tabular}{lll}
\hline Variable & Placebo & BTX-A \\
\hline CVA & 9 & 14 \\
Head injury & 8 & 4 \\
Spinal cord injury & 2 & 4 \\
Tumour & 4 & 1 \\
Cerebral palsy & 0 & 3 \\
Anoxic brain damage & 2 & 1 \\
Total number & 25 & 27 \\
Age (y) & Mean $38.8(32,16-79)$ & Mean $41.7(44,17-71)$ \\
M:F ratio & SD 16.6 & SD 17.8 \\
Duration since injury (months) & $17: 8$ & $18: 9$ \\
& Mean $43.4(20,3-228)$ & Mean $27.2(12,4-264)$ \\
Dose of toxin received (IU) & SD 53.1 & SD 49.4 \\
& Mean 210.6 (225, 40-500) & Mean $183.3(175,30-400)$ \\
Limb affected (upper:lower) & SD 127.7 (saline) & SD 101.3 \\
& 16:9 & $16: 11$ \\
\hline
\end{tabular}

Values in parentheses are medians and ranges; $\mathrm{CVA}=$ cerebrovascular accident.

Table 3 Comparison of dependent variables at baseline

\begin{tabular}{|c|c|c|}
\hline Variable & Placebo & $B T X-A$ \\
\hline Ashworth scale $(0-4)$ & $\begin{array}{l}\text { Mean } 3.0(3,2-4) \\
\text { SD } 0.7\end{array}$ & $\begin{array}{l}\text { Mean } 2.7(3,1-4) \\
\text { SD } 0.8\end{array}$ \\
\hline Range of movement (\% of full angle) & $\begin{array}{l}\text { Mean } 57.9(55.6,0-100) \\
\text { SD } 23.5\end{array}$ & $\begin{array}{l}\text { Mean } 67.6(71.4,14.3-100) \\
\text { SD } 24.6\end{array}$ \\
\hline $\begin{array}{l}\text { Rivermead gross motor function } \\
\text { scale }(0-13)\end{array}$ & $\begin{array}{l}\text { Mean } 7.4(10,0-11) \\
\text { SD } 4.1\end{array}$ & $\begin{array}{l}\text { Mean } 8.3(9,0-13) \\
\text { SD } 3.5\end{array}$ \\
\hline $\begin{array}{l}\text { Rivermead limb and trunk function } \\
(0-10)\end{array}$ & $\begin{array}{l}\text { Mean } 3.9(3,2-9) \\
\text { SD } 2.1\end{array}$ & $\begin{array}{l}\text { Mean } 5.5(4,3-10) \\
\text { SD } 3.0\end{array}$ \\
\hline $\begin{array}{l}\text { Rivermead upper limb function } \\
(0-15)\end{array}$ & $\begin{array}{l}\text { Mean } 2.2(1,0-8) \\
\text { SD } 2.8\end{array}$ & $\begin{array}{l}\text { Mean } 3.0(2,1-7) \\
\text { SD } 3.2\end{array}$ \\
\hline Subjective rating of problem $(0-7)$ & $\begin{array}{l}\text { Mean } 2.1(1,0-7) \\
\text { SD } 2.1\end{array}$ & $\begin{array}{l}\text { Mean } 1.9(2,1-7) \\
\text { SD } 1.3\end{array}$ \\
\hline
\end{tabular}

Values in parentheses are medians and ranges.

was similar 3.30 (range 1-6) but the mean size of the dose was larger 284.75 (SD 108.37) units (range 75-500 ). Doses were guided by clinical experience; table 4 provides a sample of the doses used for specific patients in the trial. A useful clinical guide to doses can be found in Mitchell et $a^{23}$

For the Ashworth scale, the Mann-Whitney test on the aggregate outcome score compares the groups postbaseline. The active treatment group had significantly better scores than the placebo group $(S=780.5, z=2.16, p<0.02)$. The ANOVA on the rank transformed Ashworth data showed that there was a significant time effect $\left(F_{4,200}=11.2, \mathrm{p}<0.0001\right)$ and a significant interaction between time and group $\left(F_{4,200}=2.6, \mathrm{p}<0.05\right)$. Inspection of fig 2 and table 5 shows that the Ashworth scores improved (became lower) for both groups from the baseline to the week 3 assessment with little further improvement thereafter. In addition, the treated group's score at week 3 was more marked than that of the placebo group. These impressions were confirmed by the contrast analyses. Across both groups, Ashworth scores were significantly improved at week 3 relative to baseline $\left(F_{1,50}=27.5, \mathrm{p}<0.0001\right)$ but subsequent successive time contrasts (week $6 v$ week 3 ; week $9 v$ week 6 ; week $12 v$ week 9) were insignificant $\left(F_{1,50}<1.5, \mathrm{p}>0.2\right.$ in every case). The differential improvement at the week 3 assessment in the treated group was confirmed by a significant interaction between group and the week $3 v$ baseline contrast $\left(F_{1,50}=10.8\right.$, $\mathrm{p}<0.002)$. This was not seen in any of the other successive time contrasts $\left(F_{1,50}<2.8, \mathrm{p}>0.1\right.$ in every case).

For the range of movement index, the aggregated outcome scores showed change in both groups but were significantly better for the treated than for the placebo group $(S=559$, $z=1.89, \mathrm{p}<0.03)$. For both groups there was an improvement in scores across time $\left(F_{4,200}=15.0\right.$ $\mathrm{p}<0.0001)$ which occurred between baseline and 3 weeks (contrast $F_{1,50}=16.8, \mathrm{p}<0.0002$ ). However, there was no interaction between group and these time effects (group by time, $\left.F_{4,200}=0.7, \mathrm{p}>0.5\right)$. This indicates that the pattern of range of movement scores over time points was the same for both groups (although the main effect of time indicated that the pattern for both groups combined was for an increase in range of movement, particularly between baseline and 3 weeks).

For the gross motor function score of the Rivermead motor assessment scale, there was no group difference in aggregated outcome scores $(S=596.5, z=1.22, \mathrm{p}>0.1)$. ANOVA showed a general change from baseline (time effect: $\left.F_{4,200}=7.6, \mathrm{p}<0.0005\right)$ but no effect of group on these changes (group by time interaction: $\left.F_{4,200}=0.9, \mathrm{p}>0.4\right)$. The analysis of gross motor function combines scores from both upper and lower limbs. Further analysis should be obtained by concentrating exclusively on the motor functions of the affected limbs. The Rivermead scores for the affected limbs were analysed separately for patients with upper and lower limb impairments. For the 32 patients with upper limb dysfunction, there was no effect of treatment group on the aggregated outcome score $(S=238, z=0.99, \mathrm{p}>0.15)$. For

Table 4 Samples of doses given for specific muscle groups and problems presenting for treatment

\begin{tabular}{|c|c|c|}
\hline Problem & Muscles & $\begin{array}{l}\text { Dose (Allergan product } \\
\text { Botox; IU) }\end{array}$ \\
\hline \multirow[t]{4}{*}{ Unable to place the foot flat on the floor } & Gastroc medial head & 100 \\
\hline & Gastroc lateral head & 100 \\
\hline & Soleus & 100 \\
\hline & Tibialis posterior & 100 (total dose 400 ) \\
\hline Extending big toe causing pain on walking & Extensor hallucis longus & 75 units \\
\hline \multirow{3}{*}{ Clawing of the 2 nd to 5 th toe with extension of big toe } & Extensor hallucis longus & 50 \\
\hline & Flexor digitorum brevis & 100 \\
\hline & Tibialis posterior & 50 (total dose 200) \\
\hline \multirow[t]{3}{*}{ Flexion of elbow when walking } & Biceps & 50 \\
\hline & Brachioradialis & 30 \\
\hline & Brachialis & 20 (total dose 100 ) \\
\hline \multirow[t]{2}{*}{ Difficulty grasping objects due to wrist flexion } & Flexor carpi ulnaris & 30 \\
\hline & Flexor carpi radialis & 50 (total dose 80 ) \\
\hline \multirow[t]{3}{*}{ Difficulty cleaning the palm of the hand and caring for finger nails } & Flexor carpi ulnaris & 75 \\
\hline & Flexor carpi radialis & 75 \\
\hline & Flexor digitorum superficialis & 75 (total dose 225) \\
\hline $\begin{array}{l}\text { Unable to extend fingers fully for functional tasks such as clicking } \\
\text { computer mouse }\end{array}$ & Flexor digitorum profundus & 30 \\
\hline
\end{tabular}



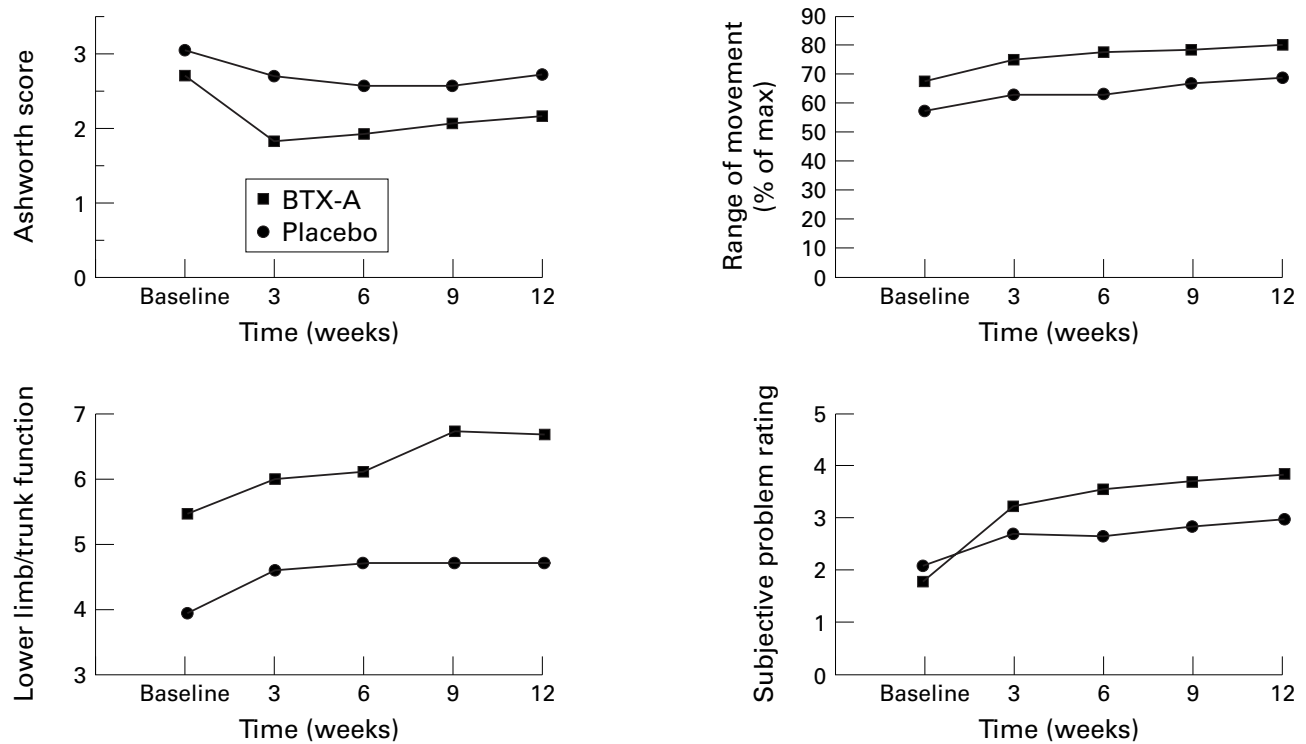

Figure 2 Mean scores of the raw data (unranked) at each time point for the key dependent variables (Ashworth, subjective rating of the problem severity, range of movement, and Rivermead motor assessment scale lower limb/trunk).

Table 5 Mean ranks on the critical dependent variables

\begin{tabular}{|c|c|c|c|c|c|c|c|c|}
\hline Variable & Group & Baseline & Week 3 & Week 6 & Week 9 & Week 12 & Aggregate* & $\begin{array}{l}\text { Contrast analysis } \\
\text { p value }\end{array}$ \\
\hline \multirow[t]{2}{*}{ Ashworth } & Placebo & 3.6 & $3.2 \varnothing$ & 2.6 & 2.7 & 2.9 & $10.3(12)$ & \multirow{10}{*}{$<0.002$} \\
\hline & BTX-A & 4.2 & $2.4 \varnothing$ & 2.5 & 2.9 & 3.0 & $7.8(6)$ & \\
\hline \multirow[t]{2}{*}{ Range of movement } & Placebo & 2.1 & 3.1 & 2.9 & 3.4 & 3.6 & $102.8(20)$ & \\
\hline & BTX-A & 2.0 & 2.9 & 3.4 & 3.4 & 3.4 & $33.8(4)$ & \\
\hline \multirow[t]{2}{*}{ Gross motor function } & Placebo & 2.8 & 2.9 & 3.0 & 3.1 & 3.3 & $30.1(40)$ & \\
\hline & BTX-A & 2.6 & 2.7 & 3.2 & 3.1 & 3.4 & $39.1(40)$ & \\
\hline \multirow[t]{2}{*}{ Motor function; upper limb $\dagger$} & Placebo & 2.5 & 3.0 & 2.9 & 3.3 & 3.2 & $11.0(6.5)$ & \\
\hline & BTX-A & 2.0 & 3.0 & 3.3 & 3.5 & 3.2 & $18.0(12.5)$ & \\
\hline \multirow[t]{2}{*}{ Motor function; limb/trunk $\ddagger$} & Placebo & 1.9 & 3.1 & 3.4 & 3.2 & 3.4 & $18.6(16)$ & \\
\hline & BTX-A & 2.2 & 3.0 & 3.0 & 3.3 & 3.5 & $25.5(21)$ & \\
\hline \multirow[t]{2}{*}{ Problem ratings } & Placebo & 2.5 & $3.0 \$$ & $2.9 \sqrt{9}$ & 3.2 & 3.3 & $11.1(10)$ & \multirow{2}{*}{$\begin{array}{l}<0.05 \\
<0.01\end{array}$} \\
\hline & BTX-A & 1.5 & $2.7 \S$ & $3.4 \rrbracket$ & 3.7 & 3.8 & $14.5(14)$ & \\
\hline
\end{tabular}

*The aggregate is the sum of the raw scores for weeks 3 to 12 inclusive. The values in the aggregate column are means with medians in parentheses.

†For patients with upper limb deficits; $\ddagger$ for patients with lower limb deficits.

\$Denotes point at which a significant change from the previous value is statistically significant as detected with ANOVA performed on the ranked data and confirmed by contrast analysis.

both treatment and control groups within this subset there was an improvement in scores across time $\left(\mathrm{F}_{4,120}=9.0, \mathrm{p}<0.0001\right)$ which occurred between baseline and the week 3 assessment (contrast $\mathrm{F}_{1,30}=13.9, \mathrm{p}<0.001$ ). There was no interaction between group and these time effects (group by time, $F_{4,120}=1.3, \mathrm{p}>0.25$; group by baseline $v$ week 3 contrast, $F_{1,30}=1.5$, $\mathrm{p}>0.2)$. The aggregated outcome scores for the 20 cases with lower limb dysfunction, were significantly better for the treated than for the placebo group $(S=71.5, z=1.72, \mathrm{p}<0.05)$. For both groups there was an improvement in scores across time $\left(F_{4,72}=6.7, \mathrm{p}<0.0001\right)$ which again occurred between baseline and the week 3 assessment (contrast $\mathrm{F}_{1,18}=9.5, \mathrm{p}<0.01$ ). However, as with the upper limb group there was no interaction between group and these time effects (group by time, $F_{4,72}=0.5, \mathrm{p}>0.5$; group by baseline $v$ week 3 contrast, $F_{1,18}=0.5, \mathrm{p}>0.4$ ).

A subset of the patients with lower limb impairments were able to complete the 10 metre timed walk test at each assessment point (nine placebo $v 11$ treated). The aggregated outcome scores did not differ significantly between the two groups $(S=108.5, z=1.03$, $\mathrm{p}>0.3)$. The time effect was significant $\left(F_{4,72}\right.$ $=4.3, \mathrm{p}<0.01)$ but the time by group interaction was not $\left(F_{4,72}=0.07, \mathrm{p}>0.5\right)$. Within the subset of patients with upper limb impairment only six were able to perform the nine hole peg test, providing insufficient data for analyses.

For the subjective ratings of problems the aggregated outcome scores of the treated group were significantly higher (better) than those of the placebo group $(S=551.5, z=2.0, \mathrm{p}<0.025)$. An ANOVA showed that both the effect of time $\left(F_{4,200}=25.4, \mathrm{p}<0.0001\right)$ and the interaction between group and time $\left(F_{4,200} 7.6, \mathrm{p}<0.0002\right)$ were significant. Inspection of fig 2 and table 4 shows that the subjective ratings improved for both groups from the baseline to the week 3 assessment with smaller improvements thereafter. In addition, the treated group's improvement between baseline and week 3, and between week 3 and week 6 , seemed greater than that of the placebo group. These impressions were confirmed by the contrast analyses. Across both groups, problem ratings showed a large change at week 3 relative to baseline $\left(F_{1,50}=22.7, \mathrm{p}<0.0001\right)$ with smaller but significant improvements from week 3 to week 6 
$\left(F_{1,50}=4.5, \mathrm{p}<0.05\right)$ and from week 6 to week 9 $\left(F_{1,50}=5.8, \mathrm{p}<0.02\right)$. Only the first two successive time contrasts interacted significantly with group (week $3 v$ baseline, $F_{1,50}=4.5, \mathrm{p}<0.05$; week $6 v$ week $\left.3, F_{1,50}=8.3, \mathrm{p}<0.01\right)$.

The rank transformation approach suggested by Conover and Iman, ${ }^{22}$ which we adopted, does not attempt to produce normal distributions (by contrast with the use of log transformations). They suggest it as a simple way to carry out non-parametric tests on a wide variety of designs.

The goal attainment scaling showed that 10 out of the 27 treated patients achieved their goal by the end of the trial (37\%) compared with $9 / 25(36 \%)$ of the placebo group. The figures for partial achievement (treated $22.2 \%$; placebo $20 \%$ ) were also very similar for the two groups.

For the variables which showed a specific treatment effect-that is, the Ashworth scale scores, subjective rating of problem severity, percentage of passive range of movement scores, and the Rivermead lower limb/trunk scores-the data at week 12 were used to determine if the effect was maintained. The Mann-Whitney test showed that sustained improvement was most marked for the subjective rating $(S=543, \mathrm{z}=2.2, \mathrm{p}<0.02)$, and the Ashworth score $(S=753, z=1.71, \mathrm{p}<0.005)$, with a trend only in the passive range of movement scores $(S=590, \mathrm{z}=1.32, \mathrm{p}<0.1)$ and Rivermead lower limb score $(S=753, z=1.71$, $\mathrm{p}<0.08$ ).

To determine the effectiveness of the blinding, the researcher responsible for assessing and measuring throughout the trial was asked to judge whether the patient had received active or placebo treatment at the end of the study. Of the 27 patients receiving active treatment, the researcher correctly identified 14 $(51.9 \%)$; of the 24 patients receiving placebo she correctly identified $13(52.0 \%)$. A $\chi^{2}$ test disclosed that there was no association between the researcher's judgments and the actual treatment received $\left(\chi^{2}=0.08, \mathrm{df}=1, \mathrm{p}>0.7\right)$ indicating successful blinding in the trial.

No side effects were recorded by any of the patients apart from pain at the time of injection, which was reported by four patients.

\section{Discussion}

The purpose of the study was to determine the effect of using EMG guided BTX-A injections within a rehabilitation programme where patients presented with specific problems resulting from focal hypertonia. Both placebo and treatment groups improved, with greater improvement in the treated group. Patients enrolled in the study were a highly selected sample in whom a potential for further improvement was identified. Recruitment involved detailed analysis of a specific problem with multidisciplinary assessment and careful follow up. All patients received advice and guidance on targeting physiotherapy to the problem and splinting advice was given where appropriate. It was not possible to control for the type of rehabilitation programme in which they were already participating.
Benefits in impairment and focal disability were investigated. The primary measure of impairment was measurement of muscle tone using the Ashworth scale. The treated group was significantly superior on their aggregate Ashworth score during treatment (combined across all postbaseline assessments). An ANOVA showed that there was a differential improvement between groups in Ashworth scores between baseline and the week 3 assessment, with the treated group showing the greater improvement. There was no significant difference between the goups in the pattern of scores over time after week 3. To determine whether the treated goup showed superior Ashworth scores to the placebo group at 12 weeks a Mann-Whitney test on the week 12 Ashworth data was performed. This showed that differences between treatment and control groups were statistically significant $(\mathrm{p}<0.005)$. The passive range of movement was recorded as a secondary measure of impairment as it is directly influenced by the degree of muscle tone. The results complement the Ashworth scores in that the greatest change in passive movement occurred at the same time as the greatest change in the recorded Ashworth scores (0-3 weeks), supporting the concept that muscle tone influences the range of movement at a joint. ${ }^{24}$

The primary measure of focal disability was the patient's or carer's self rating of problem severity. This showed a highly statistically significant response to treatment. This is probably due to the specificity of the measure. It evaluates the effect the toxin had on the presenting problem that the team identified as being potentially amenable to treatment and is therefore the most direct measure of the effect of the toxin. Furthermore, it does not rely on a fixed end point and shows that patients or carers are able to detect the subtle improvements themselves.

Of the standardised measures of focal disability, only the Rivermead motor assessment scale (leg/trunk section) showed a significant treatment effect. However, the lower limb effect is described as apparent because the treated group did show a weak trend $(p=0.13$, two tailed) to better function pretreatment. If four pretreatment measurements had been made, given the increased reliability that accrues with repeated measurement, it is possible that a significant difference between the groups might have emerged. Thus, the posttreatment effect could be a reflection of pretreatment sampling differences between the active and placebo groups. The same is true for the passive range of movement data, which also showed a weak trend $(p=0.16$, two tailed) to better range of movement pretreatment. The non-significant results of the upper limb motor scores mask clinically important improvements in individual patients. For example three patients with upper limbs treated returned to work because of improved hand function after treatment.

These results indicate that the standardised measures were insensitive to change compared with the subjective rating results. This highlights the difficulties in measuring outcome of 
such a specific and focal intervention. BTX-A has a direct effect on muscle activity and thus its effects on impairment can be recorded. Its secondary effects on the consequences of reducing hypertonia are very individualised. No single measure exists that includes all aspects of a patient's disability, many of which are unique to the particular patient. The more indirect the measure the less sensitive it is at picking up change as a result of a specific intervention. The standardised, validated measures that may not be sensitive or specific enough to identify change over time and individualised scores have limited reliability and validity but may be more responsive.

The modified goal attainment scale used showed little difference between the groups. This may indicate that this modification is not very useful as an objective outcome measure. Alternatively, because it was assessed only at 12 weeks, it might have missed group differences that tended to occur earlier, at around 6 weeks. Early goal attainment could allow for early treatment progression. We did not record whether patients achieved more than the expected goal.

Improvements made were maintained at 12 weeks for the Ashworth scale, subjective rating of problem severity, percentage of passive range of movement, and lower limb function. Further follow up would have been useful to determine whether improvement continued beyond the 3 months that is generally regarded as the period over which the toxin is effective. In the clinical management of hypertonia it is hoped that a single injection may result in sustained improvement.

The study design has made some advances on previous work. Patient selection was rigorous with the use of EMG to evaluate the degree of muscle activity that might be amenable to treatment, multidisciplinary concensus of the potential benefits from treatment, a range of outcome measures targeted at impairment, and disability inclusive of the patients perspective.

Despite these improvements limitations are still present. The heterogeneity of the group with recruitment from more than five disease groups and inclusion of both upper and lower limbs is not ideal and despite this wide recruitment criteria it was still difficult to achieve large sample numbers. The selection of outcome measures was not ideal for the upper limb group. Of the 32 patients recruited to this group, only six were able to perform the nine hole peg test, providing insufficient data to analyse. In addition, the follow up time of the study was limited and the blinding of the patients was not assessed. Perhaps the greatest limitation of the study was that it was not possible to control the rehabilitation that the patients were receiving before inclusion in the trial, it was undoubtedly varied and not standardised for amount, type, and frequency. Posttreatment intervention was controlled to a certain extent in that all patients, in both treatment and control groups, received four measurement sessions and liason with the local therapists on stretching programmes and splinting that was tailored to the individual patient's needs. However, this trial was set up to reflect the "real world" clinic environment. It could be said that this was a study of BTX-A treatment and therapy versus therapy alone.

In summary, the results show that, compared with placebo, BTX-A injections produced significantly greater improvements in Ashworth ratings (which were evident at the week 3 assessment only) and self rated problem scores (which were evident at the week 3 and week 6 assessments). Among patients with problems affecting the lower limb, there was apparent evidence of a treatment related improvement in motor function in the lower limb and trunk (based on weeks 3 to 12 combined) although it was not possible to locate the effect more precisely in time. Gross motor function indices, motor function of the upper limb (in upper limb patients), and timed 10 metre walk data (lower limb patients), did not show significant treatment effects. There was no effect of the treatment on goal attainment by the patients at the end of the trial, nor was the researcher able to detect which patients had been given the active treatment.

\section{Conclusion}

It is concluded that BTX-A has an effect on impairment and focal disability within the rehabilitation of selected patients with problems attributable to focal spasticity. BTX-A undoutedly reduces tone and increases range of movement, which may lead to improvement in focal disability although in this study this was only demonstrated in the lower limb group. The major challenge is to identifiy patients who will obtain dynamic functional improvement. Startling individual results were obscured by group analysis. There is a need to design focused trials with more sensitive measures of motor performance, accurate assessment, and treatment guidelines to ensure that those patients who have the potential to benefit from BTX-A injections are identified and have access to the treatment and follow up.

1 Lance JW. Pathophysiology of spasticity and clinical experience with baclofen. In: Feldman RG, Young RR, Koella WP, eds. Spasticity: disordered motor control. London: Year Book Medical Publishers 1980:185-204.

2 Sheean GL. Pathophysiology of spasticity. In: Sheean GL, ed. Spasticity rehabilitation. London: Churchill Communications Europe 1998:17-38.

3 Dietz V, Quintern J, Berger W. Electrophysiological studies of gait in spasticity and rigidity. Evidence that altered mechanical properties of muscle contribute to hypertonia. Brain 1981;104:431

4 O'Dwyer NJ, Ada L, Neilson PD. Spasticity and muscle contracture following stroke. Brain 1996;119:1737-49.

5 Dolley JO, Black J, Williams RS, et al. Acceptors for botulinum neurotoxin reside on motor nerve terminals and mediate its internalisation. Nature 1984;307:457-60.

6 Koman LA, Mooney JF, Smith BP, et al. Management of cerbral palsy with botulinum toxin A: report of a preliminary randomised, double-blind trial. If Paediatr Orthop 1994;14:299-303

7 Cosgrove AP, Graham HK. Botulinum toxin in the management of the lower limb in cerebral palsy. Dev Med Child Neurol 1994;36:386-96.

8 Boyd RN, Graham HK. Botulinum toxin A in the management of children with cerebral palsy: indications and outcome. Eur $\mathcal{F}$ Neurol 1997;4(suppl 2):15-22.

9 Das K, Park DM. Effect of treatment with botulinum toxin on spasticity. Postgrad Med f 1989;65:208-10.

10 Hesse S, Krajnik J, Luecke D, et al. Ankle muscle activity before and after botulinum toxin therapy for lower limb extensor spasticity in chronic hemiparetic patients. Stroke 1996;3:455-60. 
11 Dunne JW, Heye N, Dunne SL. Treatment of chronic limb spasticity with botulinum toxin A. I Neurol Neurosurg Psychiatry 1995;58:232-5.

12 Burbaud P, Wiart J, Dubos J L, et al. A randomised, double blind, placebo controlled trial of botulinum toxin in the treatment of spastic foot in hemiparetic patients. F Neurol Neurosurg Psychiatry 1996;61:265-69.

13 Simpson D M, Alexander D N, O'Brien C F, et al. Botulinum toxin type $\mathrm{A}$ in the treatment of upper extremity spasticity: a randomised, double blind, placebocontrolled trial. Neurology 1996;46:1306-10.

14 Hesse S, Reiter F, Matthias K, et al. Botulinum toxin type A and short term electrical stimulation in the treatment of upper limb flexor spasticity after stroke: a randomized, double-blind, placebo-controlled trial. Clinical Rehabilitation $1988 ; 12: 381-8$

15 Dengler R, Neyer U, Wohlfarth K, et al. Local botulinum toxin in the treatment of spastic foot drop. $f$ Neurol toxin in the treatr.

16 Reiter F, Maura D, Gabriella Cervolo M, et al. Disability changes after treatment of upper limb spasticity with botulinum toxin. Fournal of Neurological Rehabilitation 1996;10: $47-52$
17 Bohannon RW, Smith MB. Interrater reliability of a modified Ashworth scale of muscle spasticity. Phys Ther modified Ashwo

18 Norkin CC, White D J. Measurement of joint motion. A guide to goniometry. Philadelphia: GD Davies, 1995.

19 Mathiowetz V, Weber K, Volland G, et al. Reliability and validity of grip and pinch strenght evaluations. F Hand Surg (Am) 1984;9:222-6.

20 Wade D T, Wood V A, Heller A, et al. Walking after stroke. Measurement and recovery over the first 3 months. Scand 7 Rehabil Med 1987;19:25-7.

21 Lincoln NB, Leadbetter D. Assessment of motor function in stroke patients. Physiotherapy 1979;65:48-51.

22 Conover WJ, Iman R L. Rank transformations as a bridge between parametric and non-parametric statistics. The American Statistician 1981;35:124-9.

23 Mitchell B, the Spasticity Study Group. Dosing administration and a treatment algorithm for use of botulinum toxin A for adult onset spasticity. Muscle Nerve 1997; (suppl 6):S208-19.

24 Yarkony G, Sahgal V. Contractures: a major complication of craniocerebral trauma. Clin Orthop 1987;219:93-6. 